\title{
Advanced time domain wave-number sensing for structural acoustic systems. Part III. Experiments on active broadband radiation control of a simply supported plate
}

\author{
J. P. Maillard and C. R. Fuller \\ Vibration and Acoustic Laboratories, Mechanical Engineering Department, Virginia Polytechnic Institute \\ and State University, Blacksburg, Virginia 24061-0238
}

(Received 23 November 1994; revised 12 May 1995; accepted 21 June 1995)

\begin{abstract}
The present work gives further developments and experimental testing of a new time domain structural sensing technique for predicting wave-number information and acoustic radiation from vibrating structures. Most structure-borne active sound control approaches now tend to eliminate the use of microphones located in the far field by developing sensors directly mounted on the structure. In order to reduce the control authority and complexity required to minimize sound radiation, these sensors should be designed to provide error information that is solely related to the radiating part of the structural vibrations, e.g., the supersonic wave-number components in the case of planar radiators. The approach discussed in this paper is based on estimating supersonic wave-number components coupled to acoustic radiation in prescribed directions. The spatial wave-number transform is performed in real time using a set of point structural sensors with an array of filters and associated signal processing. The use of the sensing approach is experimentally demonstrated in the time domain LMS active control of broadband sound radiated from a vibrating plate. Comparisons of the control performances obtained with the wave-number sensor and error microphones in the far field show that only a few point sensors are required to provide accurate radiation information over a broad frequency range. The approach demonstrates good broadband global control of sound radiation. (c) 1995 Acoustical Society of America.
\end{abstract}

PACS numbers: $43.40 . \mathrm{Dx}, 43.40 . \mathrm{Vn}$

\section{INTRODUCTION}

Over the past few years, much research has been conducted in the area of active control applied to structure-borne sound. In active structural acoustic control (ASAC), the minimization of sound radiation is achieved by applying oscillating force inputs directly to the structure rather than by exciting the acoustic medium with loudspeakers (active noise control). The technique can often produce global far-field attenuations with relatively few actuators as compared to active noise control. In early studies, Fuller has shown both theoretically ${ }^{1}$ and experimentally ${ }^{2}$ the use of point forces as control actuators. More recent works have demonstrated the potential of multiple piezoelectric actuators to replace shakers and realize a more compact or "smart" structure. ${ }^{3}$

One of the primary concerns in active control of sound is choosing the appropriate sensor in order to provide the control system with "error" information. Error microphones located in the far field have yielded good results, since the quantity to minimize, i.e., acoustic power radiated from the structure, is directly related to the far-field pressure. However, the microphone solution is often impractical in real applications and, in an attempt to make the system more compact, the current research tends to develop radiation sensors that are mounted on the structure. One possible approach is to design structural error sensors in order to minimize the vibrations over the entire radiating surface (active vibration control). Such an approach would obviously yield sound attenuation. However it will require, in many cases, a great number of control inputs. A more practical sensing technique should take into consideration the structure/fluid interaction so that only the radiating part of the structural vibrations is observed by the sensor. The principal advantage of this approach is that it allows "modal restructuring" in the control mechanism; ${ }^{1,4}$ in some cases, the residual response is not attenuated but rather the structure is forced to behave like an inefficient radiator. As a result, the control authority and number of channels required to achieve sound attenuation is reduced. Clark and Fuller have discussed the use of polyvinylidene fluoride (PVDF) thin film modal sensors in sound radiation control for rectangular radiators below the critical frequency. ${ }^{5}$ With appropriate shapes and locations, PVDF sensors observe only those structural modes that efficiently radiate to the far field.

For planar radiators, the radiating part of the structural vibrations corresponds to the supersonic region of the wavenumber spectrum where the structural wave number is smaller than the acoustic wave number in the surrounding medium. Therefore, a possible approach is to build a wavenumber sensor that selects and estimates those supersonic wave-number components. Fuller and Burdisso previously showed the potential of a wave-number domain controller ${ }^{6}$ based on this type of error information. For implementation with broadband control algorithms, the wave-number estimate must be performed in the time domain. Following these ideas, Maillard and Fuller introduced a new sensing technique that predicts, in the time domain, supersonic wavenumber components coupled to acoustic radiation in prescribed directions. ${ }^{7,8}$ In the approach of Maillard and Fuller, 
the wave-number transform of the structural acceleration distribution is performed in real time using point sensors with an array of finite impulse response (FIR) filters and associated signal processing. The resulting error signal can be directly used as the error information in time domain control algorithms. The present work gives further development and experimental testing of the method. In particular, this paper experimentally demonstrates the use of such an approach in the active control of sound radiation.

After recalling the theoretical basis of wave-number sensing applied to acoustic radiation previously discussed in Refs. 7 and 8 , the sensor is briefly described for practical implementation. Experimental results from wave-number sensing of a simply supported plate are first discussed. The sensor output is compared to the actual wave-number component of the plate in order to evaluate the prediction accuracy. The radiation from the simply supported plate is then controlled using a three-channel feedforward control approach. The system is excited by a bandlimited white noise including the first five bending modes of the plate. Wavenumber sensing is compared to the use of error microphones. Results show the ability of the technique to replace far-field acoustic measurements and provide accurate error information over a broadband frequency range. Its use in active structural acoustic control is successfully demonstrated.

\section{THEORY}

This section briefly reviews the theoretical concepts associated with the proposed sensing technique. For more details, the reader is referred to the two previous companion papers. $^{7,8}$

\section{A. Far-field pressure and structural wave-number component}

Figure 1 shows a two-dimensional planar structure of length $L_{x}$ and width $L_{y}$ with out-of-plane displacement along the $z$ axis, $w(x, y, t)=W(x, y) e^{j \omega t}$. Time harmonic excitation is assumed here. The following results are easily extended to broadband excitations by means of Fourier transforms. The structure is mounted in an infinite baffle, i.e., $W(x, y)=0$ for $|x|>L_{x} / 2$ and $|y|>L_{y} / 2$. The symbol $\omega$ represents the angular frequency and $t$ is the continuous time. The acceleration field is given by $\ddot{w}(x, y, t)=-\omega^{2} W(x, y) e^{j \omega t}$, where $\ddot{w}$ represents the second time derivative of $w$. The pressure radiated by the structure, $p(x, y, z, t)=P(x, y, z) e^{j \omega t}$, must satisfy the three-dimensional Helmoltz equation,

$$
\left(\frac{\partial^{2}}{\partial x^{2}}+\frac{\partial^{2}}{\partial y^{2}}+\frac{\partial^{2}}{\partial z^{2}}+k_{0}^{2}\right) P(x, y, z)=0,
$$

along with the boundary condition that defines the interaction between the structure and fluid,

$$
\rho \ddot{W}(x, y)=-\frac{\partial P}{\partial z}(x, y, z) \quad \text { at } z=0,
$$

where $k_{0}=\omega / c$ denotes the acoustic wave number and $c$ is the speed of sound in the surrounding medium. The quantity $\ddot{W}(x, y)$ represents the complex amplitude of the out-ofplane acceleration distribution, i.e., $\ddot{W}(x, y)=-\omega^{2} W(x, y)$.
Defining the two-dimensional wave-number transform of the spatial distribution $f(x, y)$ as

$$
\tilde{f}\left(k_{x}, k_{y}\right)=\int_{-\infty}^{+\infty} \int_{-\infty}^{+\infty} f(x, y) e^{j k_{x} x} e^{j k_{y} y} d x d y,
$$

Eqs. (1) and (2) become, respectively, in the wave-number domain,

$$
\begin{aligned}
& \left(k_{0}^{2}-k_{x}^{2}-k_{y}^{2}+\frac{\partial^{2}}{\partial z^{2}}\right) \tilde{P}\left(k_{x}, k_{y}, z\right)=0, \\
& \rho \widetilde{\ddot{W}}\left(k_{x}, k_{y}\right)=-\frac{\partial \tilde{P}}{\partial z}\left(k_{x}, k_{y}, z\right) \quad \text { at } z=0 .
\end{aligned}
$$

Note that the sign of the forward transform in Eq. (3) is appropriately chosen to obey the Sommerfeld radiation condition. The above second-order homogeneous differential equation is solved for $\tilde{P}\left(k_{x}, k_{y}, z\right)$ as

$$
\begin{aligned}
\tilde{P}\left(k_{x}, k_{y}, z\right)= & -\frac{j \rho \tilde{W}\left(k_{x}, k_{y}\right)}{\left(k_{0}^{2}-k_{x}^{2}-k_{y}^{2}\right)^{1 / 2}} \\
& \times \exp \left[-j\left(k_{0}^{2}-k_{x}^{2}-k_{y}^{2}\right)^{1 / 2} z\right] .
\end{aligned}
$$

The term $\exp \left[+j\left(k_{0}^{2}-k_{x}^{2}-k_{y}^{2}\right)^{1 / 2} z\right]$ that represents waves going toward the structure is omitted since the acoustic medium is boundless. The single constant that remains in the solution is then found using the transformed boundary condition in Eq. (5). From Eq. (6), the transformed pressure field is seen to decrease exponentially as waves travel away from the structure for values of $k_{x}$ and $k_{y}$ such that $k_{x}^{2}+k_{y}^{2}>k_{0}^{2}$, i.e., in the subsonic wave-number region. The structure radiates in the far field only when $k_{x}^{2}+k_{y}^{2} \leqslant k_{0}^{2}$, i.e., in the supersonic wave-number region. Now, the pressure field is expressed in the spatial domain by applying the inverse wavenumber transform:

$$
\begin{aligned}
P(x, y, z)= & \frac{1}{(2 \pi)^{2}} \int_{-\infty}^{+\infty} \int_{-\infty}^{+\infty} \tilde{P}\left(k_{x}, k_{y}, z\right) e^{-j k_{x} x} \\
& \times e^{-j k_{y} y} d k_{x} d k_{y} .
\end{aligned}
$$

Junger and $\mathrm{Feit}^{9}$ showed that the above integral can be evaluated in the far field using the stationary phase approximation. Using spherical coordinates, $x=r \sin \theta \cos \phi$, $y=r \sin \theta \sin \phi$, and $z=r \cos \theta$, the points of stationary phase are found to be

$$
\bar{k}_{x}=k_{0} \sin \theta \cos \phi \quad \text { and } \bar{k}_{y}=k_{0} \sin \theta \sin \phi .
$$

The pressure field at a particular spherical coordinate now becomes a function of the acceleration wave-number transform evaluated at $\left(\bar{k}_{x}, \bar{k}_{y}\right)$ :

$$
\begin{aligned}
p(r, \theta, \phi, t)= & \frac{\rho e^{-j k_{0} r}}{2 \pi r} \\
& \times \widetilde{W}\left(k_{0} \sin \theta \cos \phi, k_{0} \sin \theta \sin \phi\right) e^{j \omega t}
\end{aligned}
$$

The main significance of the above expression can be stated as follow: For a baffled planar structure, the far-field radiation in a given direction is solely a function of the wave- 
number component evaluated at the stationary-phase wave numbers. This result motivates the design of a structural wave-number sensor.

\section{B. Estimate of the acceleration wave-number transform}

A time domain estimate of the structural out-of-plane acceleration wave-number component coupled to acoustic radiation in direction $(\theta, \phi)$ can be constructed by discretizing the spatial structural response over a grid of $N_{x}$ by $N_{y}$ measurement points equally spaced along the $x$ and $y$ axis. The continuous representation of the acceleration wave-number transform is approximated by a finite summation defined as

$$
\begin{aligned}
\widetilde{\tilde{w}}_{d}\left(k_{x}, k_{y}, t\right)= & \Delta x \Delta y \sum_{n_{x}=1}^{N_{x}} \sum_{n_{y}=1}^{N_{y}} \ddot{w}\left(x_{n_{x}}, y_{n_{y}}, t\right) \\
& \times \exp \left(j k_{x} x_{n_{x}}\right) \exp \left(j k_{y} y_{n_{y}}\right),
\end{aligned}
$$

where $\Delta x=L_{x} / N_{x}$ and $\Delta y=L_{y} / N_{y}$. Writing the stationaryphase wave numbers, $\bar{k}_{x}$ and $\vec{k}_{y}$, in terms of frequency [Eq. (8)], the discrete wave-number transform in Eq. (10) can be rewritten in terms of the frequency $\omega$ and radiation angles $(\theta, \phi)$ as

$$
\begin{aligned}
\widetilde{\tilde{w}}_{d}(w, \theta, \phi, t)= & \Delta x \Delta y \sum_{n_{x}=1}^{N_{x}} \sum_{n_{y}=1}^{N_{y}} \ddot{w}\left(x_{n_{x}}, y_{n_{y}}, t\right) \\
& \times \exp \left(j \omega \tau_{n_{x^{n}}}\right),
\end{aligned}
$$

where the time delay $\tau_{n_{x} n_{y}}$ is given by

$$
\tau_{n_{x^{n}}}=\left(x_{n_{x}} \sin \theta \cos \phi+y_{n_{y}} \sin \theta \sin \phi\right) / c .
$$

In the above expression, $\ddot{w}\left(x_{n_{x}}, y_{n_{y}}, t\right)$ represents the time domain acceleration measured at location $\left(x_{n_{x}}, y_{n_{y}}\right)$ while the exponential term is a constant magnitude and linear phase transfer function. In order to obtain causal transfer functions, i.e., transfer functions having a negative phase shift at all frequencies of interest, a constant time delay $\Delta \tau$ $=\max \left\{\tau_{n_{x} n_{y}}\right\}$ is subtracted from $\tau_{n_{x} n_{y}}$; i.e., the time delay in Eq. (11) becomes

$$
\bar{\tau}_{n_{x} n_{y}}=\tau_{n_{x} n_{y}}-\Delta \tau, \quad n_{x}=1,2, \ldots, N_{x}, \quad n_{y}=1,2, \ldots, N_{y} .
$$

The modified transfer functions can now be modeled by finite impulse response filters. Note that the discrete wavenumber component becomes $\widetilde{\tilde{w}}_{d}\left(\tilde{k}_{x}, t-\Delta \tau\right)$. It has been shown previously that this delayed error signal yields the same control performances as $\widetilde{\tilde{w}}_{d}\left(\bar{k}_{x}, t\right){ }^{7}$

It should be noted that the discrete transform of Eq. (10) allows evaluation of $\widetilde{\tilde{w}}\left(k_{x}, k_{y}, t\right)$ at any value of $\left(k_{x}, k_{y}\right)$ and thus radiation at any particular angle. The more conventional discrete Fourier transform ${ }^{10}$ is usually defined only for a discrete set of values, $n \Delta k_{x}$ and $n \Delta k_{y}$, where $\Delta k_{x}$ and $\Delta k_{y}$ represent the spatial frequency resolution along the $x$ and $y$ axes. This definition makes possible the use of fast Fourier transform algorithms in the off-line evaluation of the wavenumber transform. ${ }^{11}$ However, the FFT approach cannot be applied to real time wave-number sensing.

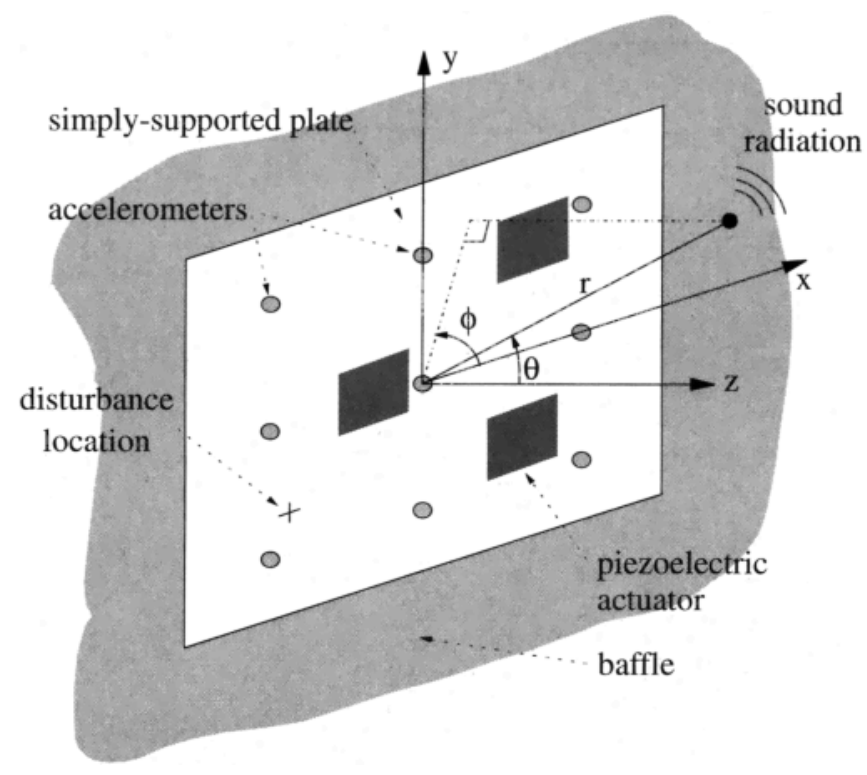

FIG. 1. Plate geometry and control arrangement.

\section{EXPERIMENTAL SETUP}

An experimental investigation of the sensing technique and ASAC implementation was performed on a simply supported plate mounted in a rigid baffle inside an anechoic chamber with a cutoff frequency of $250 \mathrm{~Hz}$. The plate is made of plain carbon steel and has dimensions $380 \times 298$ $\times 1.93 \mathrm{~mm}$. The arrangement is shown schematically in Fig. 1. Thin, flexible metal shims connect the edges of the plate to a heavy support stand to provide the simply supported boundary conditions. Table I gives the experimentally measured resonance frequencies of the first ten bending modes of the plate.

To validate the sensing method, a first set of tests was conducted to compare the wave-number components predicted by the sensing technique with the actual wave-number components of the plate over a broadband frequency range. The disturbance signal is a bandlimited random noise from 0 to $630 \mathrm{~Hz}$ which is generated by a Brüel \& Kjær 2032 spectrum analyzer and a Frequency Device 9002 low-pass filter with cutoff frequency $630 \mathrm{~Hz}$. This signal is amplified and fed to a point force shaker actuator. The shaker location is given in Table II.

The predicted wave-number component is constructed

TABLE I. Measured plate resonant frequencies.

\begin{tabular}{cc}
\hline \hline Mode $(m, n)$ & Measured frequency $(\mathbf{H z})$ \\
\hline$(1,1)$ & 87 \\
$(2,1)$ & 183 \\
$(1,2)$ & 244 \\
$(2,2)$ & 330 \\
$(3,1)$ & 343 \\
$(3,2)$ & 474 \\
$(1,3)$ & 491 \\
$(4,1)$ & 556 \\
$(2,3)$ & 571 \\
$(4,2)$ & 657 \\
\hline \hline
\end{tabular}


TABLE II. Transducer locations.

\begin{tabular}{lcc}
\hline \hline Transducer type & $x$ coordinate $(\mathrm{mm})$ & $y$ coordinate $(\mathrm{mm})$ \\
\hline disturbance (shaker) & -115 & -84 \\
control 1 (pzt) & 79 & -67.5 \\
control 2 (pzt) & 79 & 58 \\
control 3 (pzt) & -35 & -6 \\
\hline
\end{tabular}

according to the discrete formulation in Eq. (11). Figures 1 and 2 present the configuration of the wave-number sensor. Nine accelerometers are mounted on the structure to provide the structural information, i.e. $N_{x}=N_{y}=3$. Referring to Fig. 2 , the acceleration signals are sampled and passed through digital filters whose impulse responses model the transfer functions $\exp \left(j \omega \bar{\tau}_{n_{x} n_{y}}\right)$ as described in Refs. 7 and 8. The sum of the filtered signals gives the time domain estimate of the wave-number component. The wave-number sensor uses three mini B\&K accelerometers and six BBN 501 accelerometers. Six in-house developed charge amplifiers and three B\&K charge amplifiers type 2635 are used to condition the accelerometer output signals. The signal processing part of the sensing technique is implemented on a TMS320C30 digital signal processor (DSP) board installed into a host 80486based personal computer. A C code has been previously written to process up to 12 input signals and generate three output signals corresponding, respectively, to 12 structural measurements points and three wave-number components. In the following tests, the three outputs are used, thus allowing to test the sensor accuracy in three different directions simultaneously. The three estimated wave-number components were chosen to be coupled to far-field radiation at angles $\theta=-36^{\circ}, \theta=0^{\circ}$, and $\theta=36^{\circ}$ in the $x-z$ plane $\left(\phi=0^{\circ}\right)$. These three angles were found to adequately sample the pressure field in the radiation midplane. The filter impulse responses associated with each point sensor are computed analytically as described in Ref. 8. The sampling frequency was set to $2000 \mathrm{~Hz}$ and each filter had three coefficients to model the transfer functions. In order to compensate for the slight magnitude and phase differences between the dynamic response of each accelerometer and charge amplifier path, each "sensing" discrete impulse response is convolved with a "calibration" impulse response. The calibration impulse response is implemented by a FIR filter designed to model the magnitude and phase differences relative to a reference path. To this purpose, the accelerometers are initially mounted on a

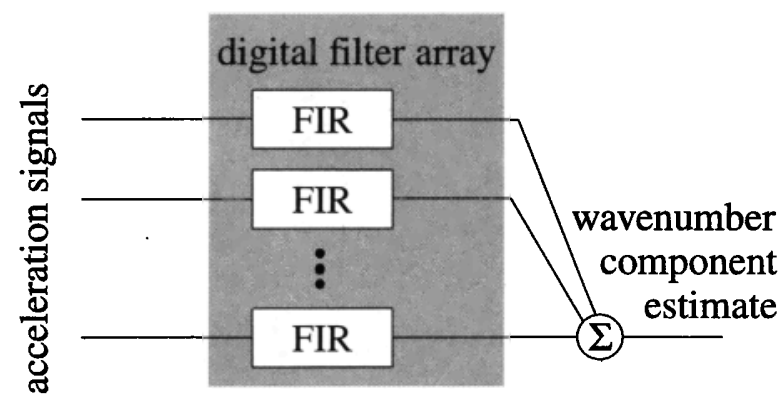

FIG. 2. Wave-number sensor block diagram. piston shaker providing constant acceleration over its surface. With the shaker being exciting over the frequency range of interest, i.e., 0-630 Hz, the outputs of the charge amplifiers are fed to the DSP board and an on-line system identification code using the LMS algorithm is implemented to update the FIR filter coefficients. Five coefficients were used for the calibration impulse response. Thus the resulting filters had seven coefficients.

The actual wave-number component of the plate is computed using the plate out-of-plane response data measured by a laser vibrometer. The laser vibrometer is mounted on a computer-controlled linear traverse that allows the measurements of the transfer function between the disturbance signal and the plate out-of-plane velocity on an eight by seven grid of points equally spaced on the plate. The plate modal amplitudes and its wave-number transform are then computed off-line as described in the Appendix. Preliminary studies on an analytical model showed that the above discretization gave almost perfect matching between continuous, i.e., the closed-form expression of the wave-number transform, and discrete representation, on the frequency range of interest. Here, the discrete representation refers to the off-line estimate of wave-number components using Eq. (10) rather than the output of the wave-number sensor.

The second set of experiments was conducted to demonstrate the use of the sensing technique in a feedforward sound radiation control approach with broadband disturbances. Three single-sided G1195 PZT piezoelectric actuators with dimension $38 \times 32 \times 0.19 \mathrm{~mm}$ are mounted on the plate as control inputs. Their center coordinates are given in Table II and they are shown schematically in Fig. 1. The disturbance input is applied through the same shaker as for the sensing tests. The low-pass filter cutoff frequency is now set to $400 \mathrm{~Hz}$ in order to include the first five bending modes of the plate. The wave-number sensor also uses the same configuration as for the sensing tests, i.e., same sampling frequency and filter coefficients. To compare the performances of the wave-number sensor with far-field pressure sensing, three error microphones are set up in the radiation field. The three microphones are located in the horizontal plane $\left(\phi=0^{\circ}\right)$ at a radius $r=1.85 \mathrm{~m}$ along the three directions of pressure estimate given above. The filtered-X version of the LMS algorithm ${ }^{12}$ is implemented on a second TMS320C30 DSP board in order to drive the three control inputs. The control system adapts the coefficients of three FIR compensators based upon the error signals, i.e., outputs of wave-number sensor or far-field error microphones, and the reference signal filtered by an estimate of the transfer functions between each one of the three control input signals and three error output signals. These nine fixed transfer functions can be modeled by infinite impulse response (IIR) filters in the case of broadband disturbances as discussed in Refs. 13 and 14. The reference signal is taken from the output of the low-pass filter, thus providing a signal coherent with the disturbance. The sampling frequency of the controller board is set to $1500 \mathrm{~Hz}$. A set of nine 35th-order IIR filter are used to model the filtered-X paths, while the three adaptive FIR compensators have a 35 coefficient impulse response. Note that a single board could implement both sens- 
TABLE III. Extra microphone locations.

\begin{tabular}{lccc}
\hline \hline & $r(\mathrm{~m})$ & $\theta(\mathrm{deg})$ & $\phi(\mathrm{deg})$ \\
\hline location 1 & 1.19 & 48 & 8 \\
location 2 & 1.32 & 10 & 0 \\
location 3 & 1.96 & 53 & -148 \\
\hline \hline
\end{tabular}

ing and control part, due to the relatively low computational load required by the wave-number sensor. However for these laboratory tests, it was more convenient to program the sensing and control on separate DSP boards. To monitor the radiated sound from the uncontrolled and controlled system, a microphone traverse located in the far-field measures the pressure field in the horizontal plane at 21 angles with a 9deg increment. Three extra fixed microphones provide pressure information out of the horizontal plane in order to confirm the global nature of the control. Their location appears in Table III. The laser vibrometer data is also used to compute the plate modal amplitudes before and after control as described in the Appendix. All the measured time domain signals are transformed into the frequency domain using a B\&K analyzer type 2032 .

\section{RESULTS}

\section{A. Wave-number sensing}

Analytical results and simulations from Refs. 7 and 8 show that only a small number of point sensors are required to estimate wave-number components for the low-order modes of a simply supported beam. The following section discusses the experimental results obtained with the simply supported plate and sensor presented earlier.

Figure 3 shows the magnitude of the actual (solid line) and predicted (dashed line) wave-number components associated with direction of radiation $\theta=36^{\circ}$. Both transfer functions are relative to the disturbance signal (output of the signal generator) and the peaks observed in the response do not always match the resonance frequencies given in Table $I$. As in standard modal analysis practice, these resonance frequen-

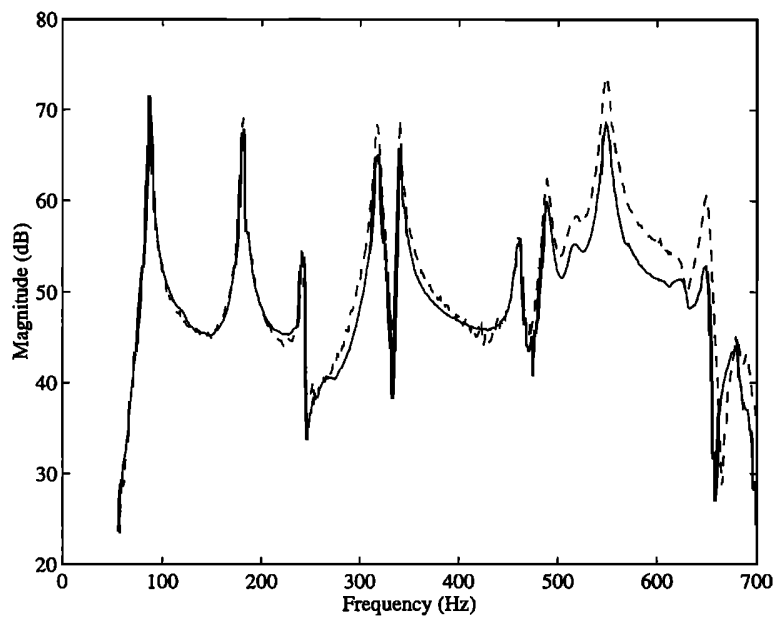

FIG. 3. Actual (—) and predicted (----) wave-number component coupled to direction of radiation $\left(\theta=36^{\circ}, \phi=0^{\circ}\right)$. cies are based on transfer functions relative to the output signal of a force transducer monitoring the input force applied by the disturbance shaker. The plot shows excellent matching between actual and predicted wave-number components up to $200 \mathrm{~Hz}$. Slight magnitude increases occur above this frequency but the variations remain within $3 \mathrm{~dB}$ over the first five modes of the plate. Above $400 \mathrm{~Hz}$, the error between actual and predicted wave-number component further increases reaching a maximum of $8 \mathrm{~dB}$ at the resonance frequency of mode $(4,2)$. The phase angle exhibits the same trends and is not shown here. Also note that similar performances were obtained in the two other directions of prediction, i.e., $\theta=-36^{\circ}$ and $\theta=0^{\circ}$. Since the radiated pressure is directly proportional to the wave-number component corresponding to the direction of radiation, the above results ensure that the sensor is also providing good broadband prediction of sound radiation as discussed later.

The variations between actual and predicted wavenumber components can be further discussed in terms of aliasing due to the discrete integration scheme of the wavenumber transform. A one-dimensional structure is considered here for simplicity. As discussed by Fahy, ${ }^{15}$ the $p$ th bending mode presents a main peak in the wave-number domain at $k_{x}= \pm p \pi / L$. Hence significant aliasing will occur at the corresponding resonance frequency if the Nyquist wave number, $K_{s}=\pi / \Delta x\left(\Delta x=L / N_{d}\right.$ being the spatial sampling period), is smaller than $p \pi / L$ or $N_{d}<p$. Moreover, since the structure is finite, the main peak obtained at a resonance frequency will be scattered into a continuum of smaller wave-number components extending up to infinity. Therefore, some aliasing will also occur for modes where $N_{d}>p$. In order to reduce aliasing errors, it is necessary to filter out the wave-number components that are higher than the Nyquist wave number. No sensor design has been proposed at this time to achieve spatial wave-number filtering. Such a sensor should convolve the spatial distribution of the structural response with the appropriate impulse response, whose wave-number transform has the characteristics of a low-pass filter. It should be noted that distributed sensors, such as PVDF films, perform spatial weighting of the structural response, i.e., a multiplication rather than a convolution product. Discussion of aliasing and its effect on the discrete wave-number transform and aliasing are also given in Ref. 7.

To further validate the wave-number sensing technique, it is of interest to study how the wave-number information relates to the actual radiated pressure. In theory, wavenumber components coupled to a given direction of radiation are related to the far-field pressure by a constant magnitude and linear phase factor as expressed in Eq. (9). Figure 4 shows the magnitude of the transfer function between the signal generator and the measured actual wave-number component coupled to direction $\theta=36^{\circ}$ along with the magnitude of the measured transfer function between the signal generator and the traverse microphone output located along the same direction. Note that a scaling factor has been introduced to facilitate the comparison. Very good agreement between wave-number information and measured radiated pressure is obtained at practically all frequencies. The important variation noticed at the resonance frequency of mode $(1,2)$ of 


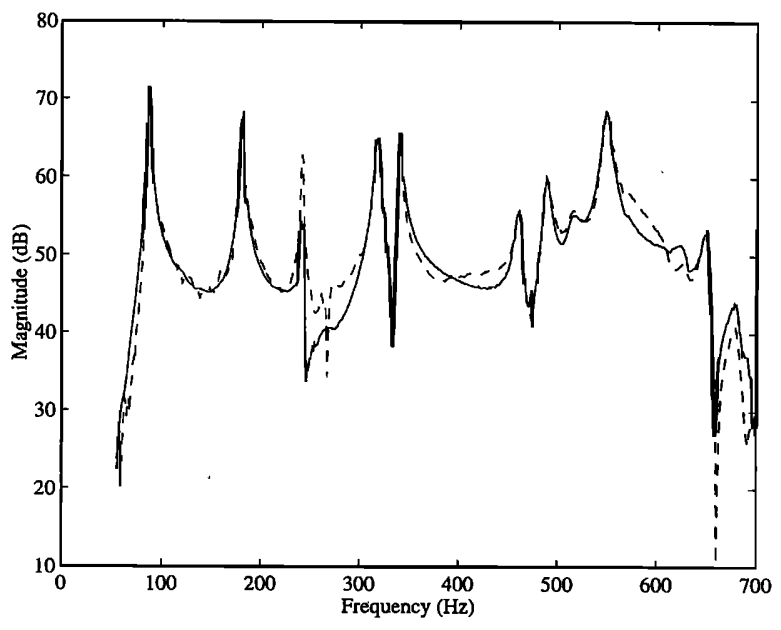

FIG. 4. Actual (-) wave-number component and measured (----) pressure in direction of radiation $\left(\theta=36^{\circ}, \phi=0^{\circ}\right)$.

the plate, $244 \mathrm{~Hz}$, is mainly due to $60-\mathrm{Hz}$ noise in the microphone charge amplifier used for the measurements. Very poor coherence (below 0.4) was noticed at this frequency. It should also be noted that the even modes along the $y$ direction, i.e., modes $(1,2),(2,2)$, and $(3,2)$, do not exhibit the expected zero sound-pressure level in the horizontal $x-z$ plane. Due to imperfections in the boundary conditions as well as the discontinuities introduced by the piezoelectric patches and the disturbance shaker, the mode shapes of the plate are not perfectly symmetric with respect to the $x$ and $y$ axes. Hence, the velocity distribution along the $y$ direction does not perfectly cancel to give zero far-field pressure in the horizontal plane.

\section{B. Radiation control}

The second set of experiments described in this paper implements wave-number sensing in the radiation control of the plate presented earlier. As described in part II, the control approach uses a three channel filtered-X LMS algorithm and the wave-number sensor provides an estimate of the accel-

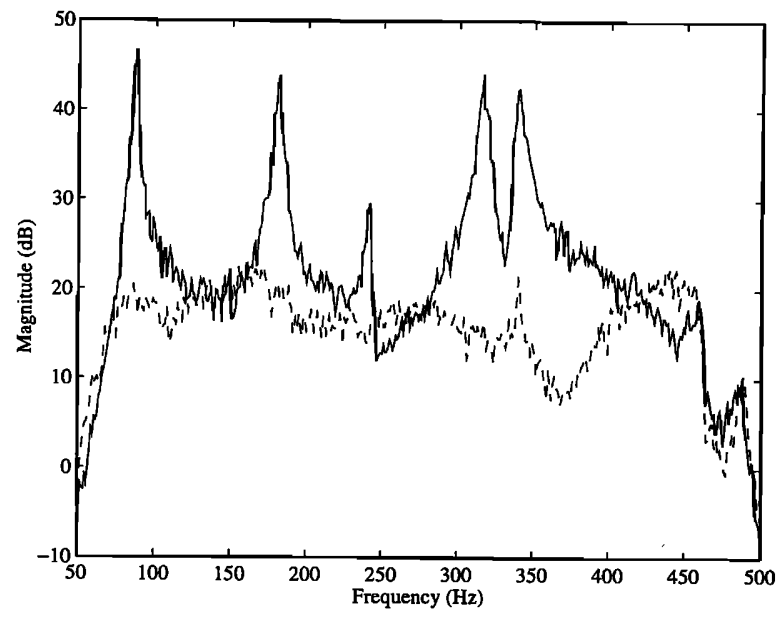

FIG. 5. Auto-spectrum of the third wave-number sensor error signal $(\theta$ $\left.=36^{\circ}, \phi=0^{\circ}\right)$ before $(\longrightarrow)$ and after (----) control.

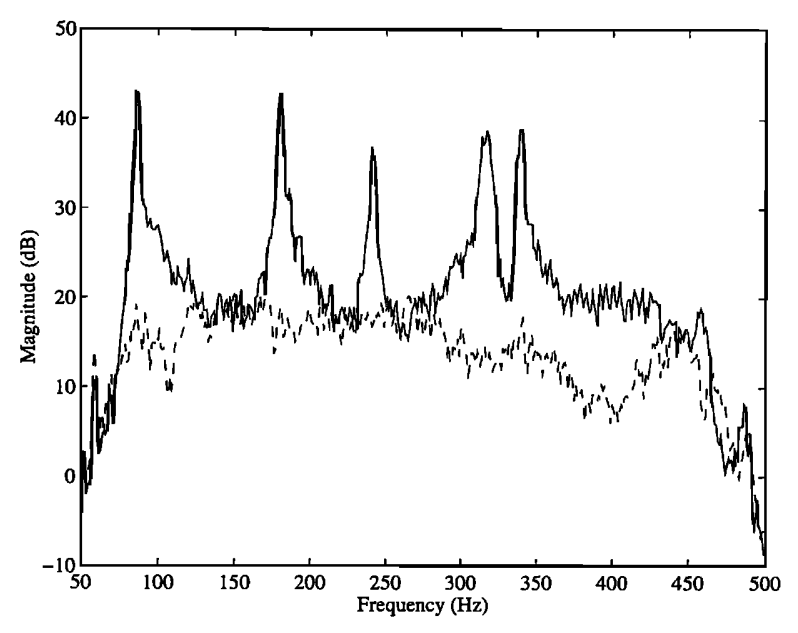

FIG. 6. Auto-spectrum of the third microphone error signal $\left(\theta=36^{\circ}, \phi=0^{\circ}\right)$ before (-

eration wave-number components coupled to radiation in directions $\theta=-36^{\circ}, \theta=0^{\circ}$, and $\theta=36^{\circ}$. Three microphones located in the far field at the same angles in the horizontal plane are also used as error signals in order to compare the relative performances of both types of sensing.

Figure 5 shows the experimentally measured autospectrum of the third error signal (wave-number component estimate coupled to radiation at angle $\theta=36^{\circ}$ ) for the uncontrolled (solid line) and controlled (dashed line) system using wave-number sensing as described above. Significant reduction is achieved across the entire bandwidth. The radiation for each one of the five modes is attenuated and the residual response does not show any clear resonance behavior, resulting in a rather flat frequency content. The same trends were measured for the first $\left(\theta=-36^{\circ}\right)$ and second $\left(\theta=0^{\circ}\right)$ error signals. The total sound-pressure level attenuation of the three error signals was calculated by integrating the three auto-spectra over $10-600 \mathrm{~Hz}$. Reduction levels of $12,12.8$, and $13.5 \mathrm{~dB}$ were achieved, respectively. When replacing the wave-number sensor outputs by the three error microphones, similar performances are obtained in terms of error signals. Figure 6 shows the auto-spectrum of the third microphone error signal $\left(\theta=36^{\circ}\right)$ before and after control. Again, attenuation is achieved over the entire bandwidth. In this case, the

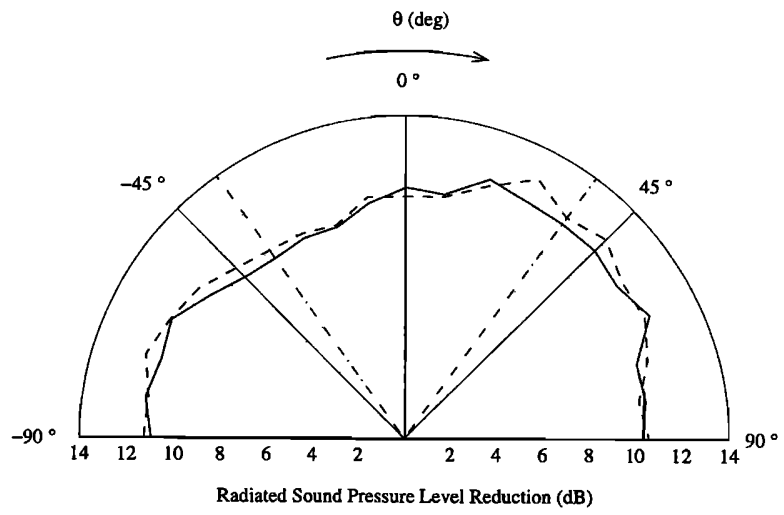

FIG. 7. Total sound-presssure level reduction integrated over $10-600 \mathrm{~Hz}$ using wave-number sensor (_- ) and error microphones (-----). 
TABLE IV. Total sound-pressure level reduction integrated over 10-600 Hz.

\begin{tabular}{lccc}
\hline \hline SPL reduction in $\mathrm{dB}$ & Location 1 & Location 2 & Location 3 \\
\hline wave-number sensing & 11.8 & 9.6 & 10.3 \\
error microphones & 12.4 & 10.6 & 10.6 \\
\hline \hline
\end{tabular}

total reduction levels for the three microphones are $9.3,10.4$, and $12.6 \mathrm{~dB}$, respectively.

In order to estimate the overall performances of both control systems using wave-number sensing or pressure measurements, far-field pressure auto-spectra are measured in the horizontal plane for $-90^{\circ} \leqslant \theta \leqslant 90^{\circ}$ and at three "random" locations out of the horizontal plane. Figure 7 shows the reduction in decibels integrated over the $10-$ to $600-\mathrm{Hz}$ bandwidth and measured in the horizontal plane. The solid line corresponds to the use of the wave-number sensor and the dashed line represents the performances obtained with error microphones. The three dashed-dotted straight lines show the three direction of minimization. The reduction obtained at the three extra locations is shown in Table IV and confirm the global nature of the control. The two sensing methods yield almost the same performances. Between 10- and 12-dB reduction is obtained at all angles and similar results can be found at the three extra locations which were out of the traverse plane. Thus the wave-number sensor appears to be fully able to replace the three error microphones in the far field.

To further compare the performances of wave-number sensing versus the use of error microphones, the system structural and acoustic response is now studied at three distinct frequencies. The plate modal amplitudes are computed at 244,305 , and $110 \mathrm{~Hz}$ from the measured broadband data (laser vibrometer), as outlined in the Appendix. Results are shown in Figs. 8, 10, and 12, respectively. For each of the above frequencies, the corresponding far-field directivity pattern obtained from the traverse microphone measurements is also presented in Figs. 9, 11, and 13, respectively.

At $244 \mathrm{~Hz}$, the plate is on resonance of the mode $(1,2)$.

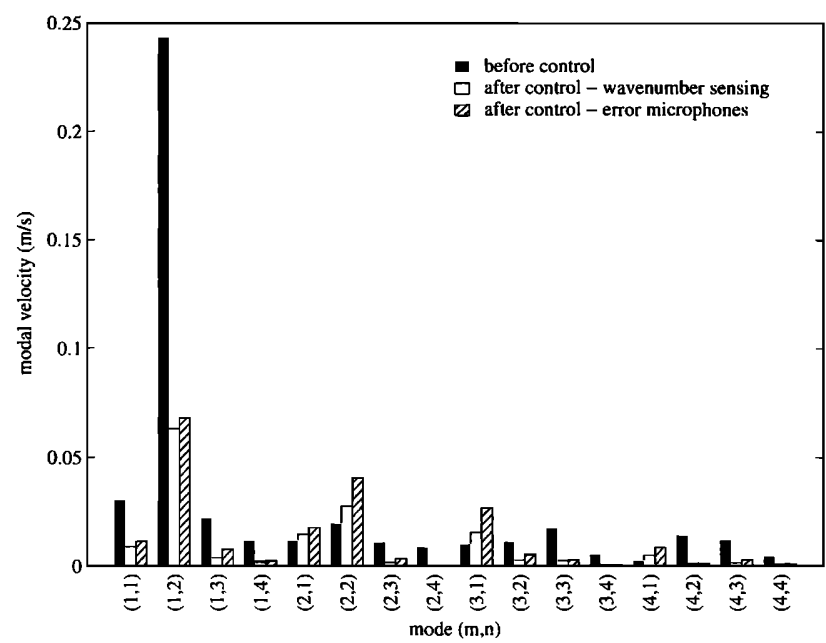

FIG. 8. Velocity modal amplitudes at $244 \mathrm{~Hz}$ before and after control using wave-number sensor and error microphones.

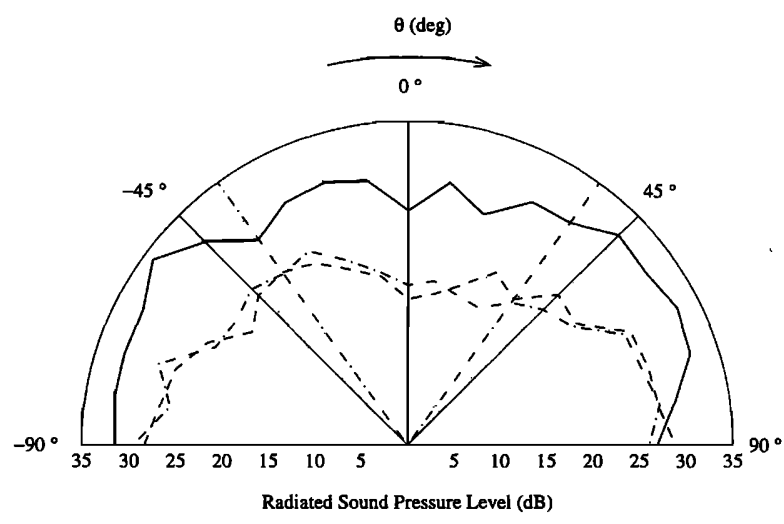

FIG. 9. Far-field sound-pressure level at $244 \mathrm{~Hz}$ before (_- _ ) and after control using wave-number sensor (----) and error microphones (-*-.-).

On-resonance cases yield better accuracy for the wavenumber sensor than off-resonance cases as shown in Fig. 3. As a result, the plate modal response after control for $244 \mathrm{~Hz}$ (Fig. 8) shows almost the same characteristics for both wavenumber and microphone sensing methods. As expected, the far-field pressure in the horizontal plane also exhibits the same behavior whether wave-number or pressure sensing is used.

Off-resonance, the sensor accuracy deteriorates and from the increased modal complexity, the control system becomes more sensitive to small variations in the error signal. As shown in Fig. 10, the modal amplitudes after control using the wave-number sensor differ slightly from the response using pressure sensing. However the variations in the error signals remain small and both set of modal amplitudes follow the same tendency. Moreover the variations do not significantly affect the far-field pressure as seen in Fig. 11. For the second off-resonance case presented here $(110 \mathrm{~Hz})$, both methods reduced mode $(1,1)$ to about the same level. However the amplitude of mode $(2,1)$ increases much more with the error microphones. In addition to the reasons given above, the far-field error measurements also differ from the estimated wave-number components due to the presence of reflected sound waves. At this frequency, the chamber is no

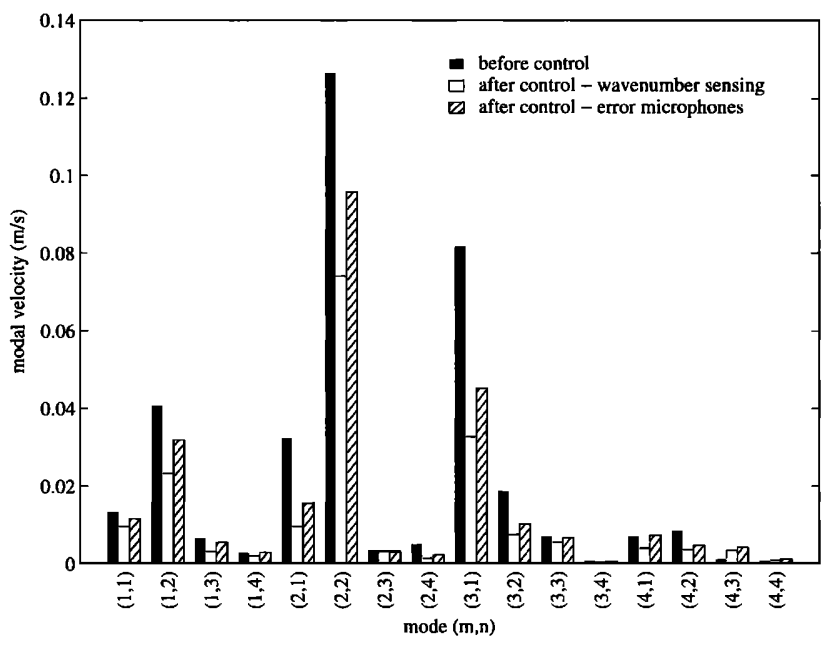

FIG. 10. Velocity modal amplitudes at $305 \mathrm{~Hz}$ before and after control using wave-number sensor and error microphones. 


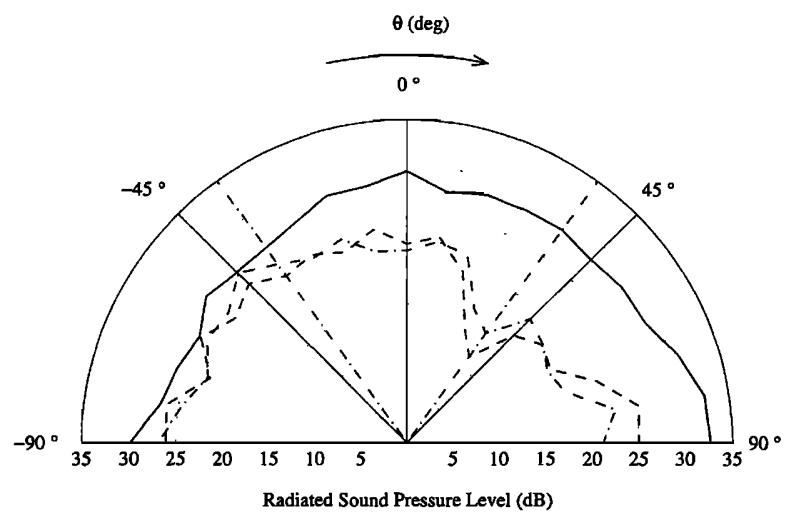

FIG. 11. Far-field sound-pressure level at $305 \mathrm{~Hz}$ before (- $\longrightarrow$ ) and after control using wave-number sensor (----) and error microphones (-*-·-).

longer perfectly anechoic. As a result, the measured sound pressure is perturbed by reflections from the chamber walls while the output of the wave-number sensor remains unaffected by the surrounding sound field. As expected, the error microphones do a better job in reducing the sound pressure in the far field. The microphones measure the free field as well as the reflected field. On the other hand, the wavenumber sensor only observed the free field, thus resulting in poorer performances. The above results present a good example of modal restructuring. ${ }^{4}$ The controlled modal amplitude of mode $(2,1)$ increases but the total structural response still has a lower radiation efficiency resulting in far field pressure reduction.

\section{CONCLUSIONS}

Broadband structural wave-number sensing has been experimentally demonstrated in the case of a baffled simply supported vibrating plate. The main significance of the approach is its ability to estimate, in the time domain, supersonic wave-number components coupled to prescribed directions of radiation over a broadband frequency range. Provided the sensor is accurate enough, it can completely replace the use of error microphones in the case of baffled

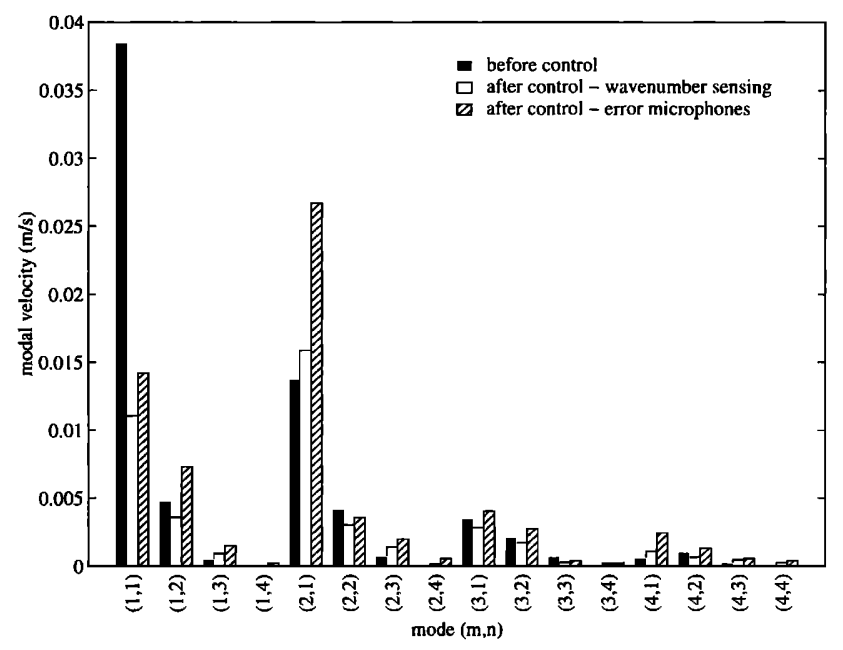

FIG. 12. Velocity modal amplitudes at $110 \mathrm{~Hz}$ before and after control using wave-number sensor and error microphones.

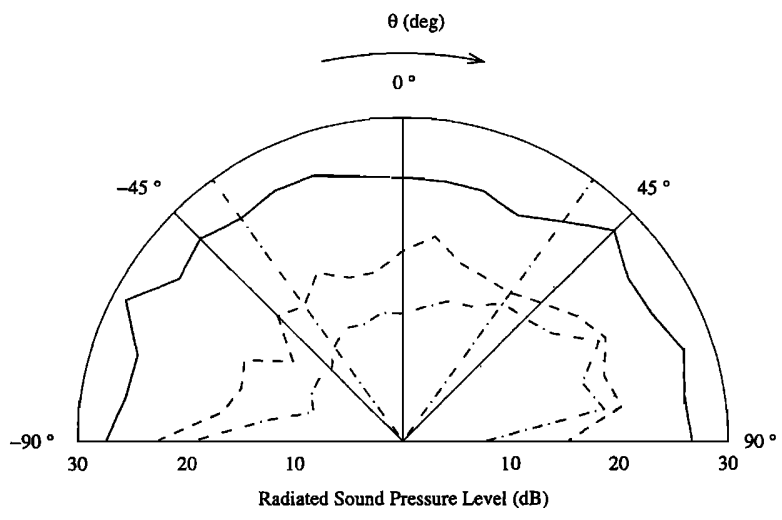

FIG. 13. Far-field sound-pressure level at $110 \mathrm{~Hz}$ before (—) and after control using wave-number sensor (----) and error microphones (-·-•-).

planar structures. The available bandwidth is directly related to the sensor complexity, i.e., number of measurement points and order of the FIR filters. When observing the low-order structural modes, comparison of predicted and actual wavenumber information shows that only a few point sensors are required to build a fairly accurate integration scheme of the wave-number transform. In order to improve the accuracy of the sensor, the use of spatially convolving sensors should be considered to provide antialiasing filters in the wave-number domain.

When applied to radiation control, wave-number sensing yields the same levels of attenuation as error microphones in the far field. By observing the radiating part of the structural vibrations (supersonic wave-number components), wavenumber sensing provides a more selective error information compared to other structural sensing methods. As a result, the control authority needed to reach a given level of sound reduction is decreased and thus, the wave-number approach results in improved performances and efficiency. Moreover, it can be of special interest when directional rather than global control is needed. Sensing radiation in prescribed directions rather than over the entire surrounding medium also reduces control authority.

The present approach is only valid in the case of baffled planar radiators. For more complex geometries and nonbaffled structures, a different type of integration scheme must be derived to account for the diffraction terms. This is the topic of future investigations.

\section{ACKNOWLEDGMENTS}

The author gratefully acknowledges the support of this work by the Office of Naval Research under Grant No. ONR-N00014-92-j-1170, Dr. Kam Ng, Technical Monitor.

\section{APPENDIX}

The evaluation of the plate modal amplitudes and wavenumber transform from the out-of-plane velocity measurements is briefly outlined. The plate out-of-plane velocity $\dot{w}(x, y, t)$ is expressed using the assumed modes method as 


$$
\begin{aligned}
\dot{w}(x, y, t) & =\sum_{m=1}^{\infty} \sum_{n=1}^{\infty} \dot{W}_{m n} \phi_{m}(x) \phi_{n}(y) e^{j \omega t} \\
& =j \omega W(x, y) e^{j \omega t},
\end{aligned}
$$

where $\dot{W}_{m n}$ is the complex modal amplitude of mode $(m, n)$. Assuming the plate is effectively subject to simply supported boundary conditions, the mode shapes along the $x$ and $y$ directions are expressed in the coordinate system of Fig. 1 as

$$
\phi_{m}(x)=\sin \left[\gamma_{m}\left(x+L_{x} / 2\right)\right]
$$

and

$$
\phi_{n}(y)=\sin \left[\gamma_{n}\left(y+L_{y} / 2\right)\right],
$$

respectively, where $\gamma_{m}=m \pi / L_{x}$ and $\gamma_{n}=n \pi / L_{y}$. Equation (A1) can be written for $N$ different locations $\left(x_{i}, y_{i}\right)$, $i=1,2, \ldots, N$. Truncating the modal summation to include the first $N$ modal amplitudes, the resulting set of equations is expressed as a linear system of the form

$$
\mathbf{S}\left\{\dot{\mathbf{W}}_{\mathbf{m n}}\right\}=\{\dot{\mathbf{w}}\} .
$$

The column vector $\dot{\mathbf{W}}_{\mathrm{mn}}$ contains the first $N$ velocity modal amplitudes $\dot{W}_{m_{j} n_{j}}, j=1,2, \ldots, N$, included in the summation of Eq. (A1). The column vector $\dot{w}$ contains the $N$ measured transfer functions between a reference signal, e.g., signal generator, and the plate out-of-plate velocity at locations $\left(x_{i}, y_{i}\right)$. The square matrix $\mathbf{S}$ is given by

$$
S_{i j}=\phi_{m_{j}}\left(x_{i}\right) \phi_{n_{j}}\left(y_{i}\right), \quad i=1,2, \ldots, N, j=1,2, \ldots, N .
$$

Since there is only a finite number of measurement points, spatial aliasing occurs in the modal decomposition. Using $N$ measurement points, only $N$ modal amplitudes can be resolved. Therefore, the response of the higher-order modes must be assumed negligible. The 8 by 7 grid used here proved to be sufficient to estimate the plate modal response at least up to $700 \mathrm{~Hz}$. This can be checked by observing the roll off in the amplitudes of the high-order modes.

After solving the linear system in Eq. (A3) for the modal amplitudes $\dot{W}_{m_{j} n_{j}}$, the wave-number transform of the out-ofplane acceleration is obtained as

$$
\widetilde{W}\left(k_{x}, k_{y}\right)=j \omega \sum_{j=1}^{N} \dot{W}_{m_{j} n_{j}} \xi_{m_{j} n_{j}}\left(k_{x}, k_{y}\right),
$$

where $\xi_{m_{j} n_{j}}\left(k_{x}, k_{y}\right)$ represents the wave-number transform of the eigenfunction of mode $\left(m_{j}, n_{j}\right)$. A closed-form expression is given as

$$
\xi_{m_{j} n_{j}}\left(k_{x}, k_{y}\right)=\tilde{\phi}_{m_{j}}\left(k_{x}\right) \tilde{\phi}_{n_{j}}\left(k_{y}\right),
$$

where

$$
\begin{aligned}
\tilde{\phi}_{m_{j}}(\gamma)= & \frac{2 \gamma_{m_{j}}}{\left(\gamma_{m_{j}}^{2}-\gamma^{2}\right)} \\
& \times\left\{\begin{array}{l}
\cos \left(\gamma L_{x} / 2\right), \text { for } m \text { odd, } \\
-j \sin \left(\gamma L_{x} / 2\right), \text { for } m \text { even, for } \gamma_{m_{j}} \neq \gamma,
\end{array}\right. \\
= & j L_{x} / 2 \exp \left(-j \gamma L_{x} / 2\right), \text { for } \gamma_{m_{j}}=\gamma, \quad \text { (A7) }
\end{aligned}
$$

and

$$
\begin{aligned}
& \tilde{\phi}_{n_{j}}(\gamma)=\frac{2 \gamma_{n_{j}}}{\left(\gamma_{n_{j}}^{2}-\gamma^{2}\right)} \\
& \times\left\{\begin{array}{l}
\cos \left(\gamma L_{y} / 2\right), \text { for } n \text { odd, } \\
-j \sin \left(\gamma L_{y} / 2\right), \quad \text { for } n \text { even, for } \gamma_{n_{j}} \neq \gamma,
\end{array}\right. \\
& =j L_{y} / 2 \exp \left(-j \gamma L_{y} / 2\right), \text { for } \gamma_{n_{j}}=\gamma \text {. }
\end{aligned}
$$

${ }^{1}$ C. R. Fuller, "Active control of sound transmission/radiation from elastic plates by vibration inputs. I. Analysis," J. Sound Vib. 136, 1-15 (1990). ${ }^{2}$ C. R. Fuller, R. J. Silcox, V. L. Metcalf, and D. E. Brown, "Experiments on structural control of sound transmitted through an elastic plate," in Proc. Am. Control Conf. 136, 2079-2089 (1989).

${ }^{3}$ B. K. Wada, J. L. Fanson, and E. F. Crawley, "Adaptive structures," J. Intelligent Mater. Syst. Struct. 1 (2), 157-174 (1990).

${ }^{4}$ R. L. Clark and C. R. Fuller, "Control of sound radiation with adaptive structures," J. Intelligent Mater. Syst. Struct. 2, 431-452 (1991).

${ }^{5}$ R. L. Clark and C. R. Fuller, "Modal sensing of efficient radiators with PVDF distributed sensors in active structural acoustic approaches," $\mathbf{J}$. Acoust. Soc. Am. 91, 3321-3329 (1992).

${ }^{6}$ C. R. Fuller and R. A. Burdisso, "A wavenumber domain approach to active control of structure-borne sound," J. Sound Vib. 148 (2), 335-360 (1991).

${ }^{7}$ J. P. Maillard and C. R. Fuller, "Advanced time domain wave-number sensing for structural acoustic systems. I. Theory and design," J. Acoust. Soc. Am. 95, 3252-3261 (1994).

${ }^{8}$ J. P. Maillard and C. R. Fuller, "Advanced time domain wave-number sensing for structural acoustic systems. II. Active radiation control of a simply supported beam," J. Acoust. Soc. Am. 95, 3262-3272 (1994).

${ }^{9}$ M. C. Junger and D. Feit, Sound, Structures and Their Interaction (MIT, Boston, 1986).

${ }^{10}$ M. Bellanger, Digital Processing of Signals: Theory and Practice (Wiley, New York, 1988), 2nd ed.

${ }^{11}$ E. G. Williams and J. D. Maynard, "Numerical evaluation of the Rayleigh integral for planar radiators using the FFT," J. Acoust. Soc. Am. 72, 2020-2030 (1982)

${ }^{12}$ S. J. Elliott, I. M. Stothers, and P. A. Nelson, "A multiple error LMS algorithm and its application to the active control of sound and vibration," IEEE Trans. Acoust. Speech Signal Process. ASSP-35, 1423-1434 (1987).

${ }^{13}$ J. S. Vipperman, R. A. Burdisso, and C. R. Fuller, "Active control of broadband structural vibrations using the LMS adaptive algorithm," $\mathrm{J}$. Sound Vib. 166 (2), 283-299 (1993).

${ }^{14}$ J. P. Smith, C. R. Fuller, and R. A. Burdisso, "Control of broadband radiated sound with adaptive structures," in Proceedings of the SPIE 1993 North American Conference on Smart Structures and Materials, 31 Jan.-4 Feb., Albuquerque, New Mexico, 1993.

${ }^{15}$ F. J. Fahy, Sound and Structural Vibration (Academic, London, 1985). 